\title{
Recommendations for the National Institute for Neurologic Disorders and Stroke spinal cord injury common data elements for children and youth with SCI
}

\author{
MJ Mulcahey ${ }^{1}$, LC Vogel ${ }^{2}$, M Sheikh ${ }^{3}$, JC Arango-Lasprilla ${ }^{4}$, M Augutis ${ }^{5}$, E Garner ${ }^{6}$, EM Hagen ${ }^{7}$, LB Jakeman ${ }^{8}$, \\ E Kelly ${ }^{2}$, R Martin ${ }^{9}$, J Odenkirchen ${ }^{8}$, A Scheel-Sailer ${ }^{10}$, J Schottler ${ }^{2}$, H Taylor ${ }^{11}$, CC Thielen ${ }^{1}$ and K Zebracki ${ }^{2}$
}

Study design: In 2014, the adult spinal cord injury (SCI) common data element (CDE) recommendations were made available. This project was a review of the adult SCI CDE for relevance to children and youth with $\mathrm{SCl}$.

Objectives: The objective of this study was to review the National Institute of Neurologic Disorders and Stroke (NINDS) adult SCI CDEs for relevance to children and youth with $\mathrm{SCl}$.

Setting: International.

Methods: The pediatric working group consisted of international members with varied fields of expertise related to pediatric SCl. The group convened biweekly meetings for 6 months in 2015. All of the adult SCI CDEs were reviewed, evaluated and modified/created for four age groups: 0-5 years, 6-12 years, 13-15 years and 16-18 years. Whenever possible, results of published research studies were used to guide recommendations. In the absence of empirical support, grey literature and international content expert consensus were garnered. Existing pediatric NINDS CDEs and new CDEs were developed in areas where adult recommendations were not appropriate. After internal working group review of domain recommendations, these pediatric CDEs were vetted during a public review from November through December 2015.

Results: Version 1.0 of the pediatric SCI CDEs was posted in February 2016.

Conclusion: The pediatric SCI CDEs are incorporated directly into the NINDS SCI CDE sets and can be found at https:// commondataelements. ninds.nih.gov.

Spinal Cord (2017) 55, 331-340; doi:10.1038/sc.2016.139; published online 15 November 2016

\section{INTRODUCTION}

Efforts are underway across the globe to develop standards for data collection and data sharing in clinical studies to maximize the value of research and to take advantage of rapid developments in use of data science for knowledge translation. In this context, the National Institute of Neurologic Disorders and Stroke (NINDS) of the National Institutes of Health (NIH) has been working since 2006 on a common data element $(\mathrm{CDE})$ project $^{1}$ that has resulted in the development of CDEs for use across neurological conditions and 18 disease-specific areas. $^{2}$ The purposes of the CDE structures are to create standards for the collection of data across studies; to facilitate participation in research by reducing data collection burden and increasing access to data collection case report forms (CRFs); and to improve data quality and cost efficiency across NINDS-funded clinical studies (1).

The adult spinal cord injury (SCI) CDEs were developed in $2014^{3,4}$ to provide community-driven recommendations for standard elements and instruments that could be used in studies spanning the wide breadth of clinical SCI research. Given the tremendous challenges that are encountered in SCI clinical trials, ${ }^{5-7}$ the CDE structure can provide benefit to researchers by reducing time and costs associated with protocol development and achieving greater consistency when applicable across performance sites. By enabling the pooling of data, CDEs can also facilitate leveraging of individual studies to be combined to achieve a broader impact. Harmonization of variable names and data structures between the NINDS SCI CDE project and the International SCI Data Set initiative ${ }^{8}$ has created synergy and enabled interoperability between the data that are collected during routine care (for example, International Basic SCI Data Sets) and those that are collected for clinical research studies, including the International Extended SCI Data Sets and the NINDS CDEs. This harmonization has the potential to create linkages between practice and research, inform quality management projects, integrate outcome parameters and measurement into clinical routines and result in a greater likelihood that research will be translated into clinical practice.

Although children represent a smaller segment of the SCI population, management of youth with SCI and interest in their long-term

${ }^{1}$ Department of Occupational Therapy, Jefferson College of Health Professions, Thomas Jefferson University, Philadelphia, PA, USA; ${ }^{2}$ Shriners Hospitals for Children, Chicago, IL, USA; ${ }^{3}$ The Emmes Corporation, Rockville, MD, USA; ${ }^{4}$ BioCruces Health Research Institute, Cruces University Hospital, Basque Foundation for Science, Bizkaia, Spain; ${ }^{5}$ Karolinska Institutet, Stockholm, Sweden; ${ }^{6}$ Austin Health, Melbourne, Victoria, Australia; ${ }^{7}$ Regional Hospital of Viborg, Viborg, Denmark; ${ }^{8} \mathrm{NIH} / \mathrm{NINDS}$ Neuroscience Center, Bethesda, MD, USA; ${ }^{9}$ Kennedy Krieger Institute, Baltimore, MD, USA; ${ }^{10}$ Swiss Paraplegic Center, Nottwil, Switzerland and ${ }^{11}$ TIRR Memorial Hermann Rehabilitation and Research \& University of Texas Health Science Center, Houston, TX, USA

Correspondence: Dr MJ Mulcahey, Department of Occupational Therapy, Jefferson College of Health Professions, Thomas Jefferson University, 901 Walnut Street, 6th Floor (642), Philadelphia, PA 19107, USA.

E-mail: Maryjane.mulcahey@jefferson.edu

Received 24 May 2016; accepted 22 August 2016; published online 15 November 2016 
Table 1 Three exceptions to the age-group rule

\begin{tabular}{ll}
\hline Exceptions to the four age groups & Rationale \\
\hline Children $\leqslant 3$ years & $\begin{array}{l}\text { Because of substantial differences in development of infants and babies } \leqslant 3 \text { years of age, age specification for some CDEs } \\
\text { were coded in 'age in months' for children } \leqslant 3 \text { years of age } \\
\text { Standard diagnostic tests }\end{array}$ \\
$\begin{array}{l}\text { Clinical practice guidelines and protocols for several diagnostic tests were examined for age guidelines. Examples include } \\
\text { diagnostic imaging, electrodiagnostics, pulmonary function tests and urodynamics. When ages were specified on clinical } \\
\text { practice guidelines, they were used for determining relevance. }\end{array}$ \\
$\begin{array}{l}\text { Research literature in the form of psychometric studies was used for age guidelines for all standardized measures and } \\
\text { instruments }\end{array}$ \\
$\begin{array}{l}\text { outcome instruments. Recommendations for outcomes instruments were based on reliability studies that were available to } \\
\text { extrapores age or that were designed to define lower age limits. }\end{array}$
\end{tabular}

outcomes have received increased attention over the past decade. ${ }^{9}$ The impact of SCI and potential interventions on function, activity and participation are complicated by ongoing physical, social and emotional development of the child and adolescent. As they are injured at a young age and live longer with injury, children are also more susceptible to long-term complications. ${ }^{10}$ Thus, emphasis on longitudinal monitoring may be even more essential for the prevention of secondary health complications and promotion of healthy living. Studies that enroll children and youth with SCI not only experience similar challenges as seen in adult clinical research studies but are also complicated by various factors, including the lack of empirical evidence on the natural history of pediatric SCI; the requirement for multicenter engagement (due to no one center having sufficient pediatric participants); the void in psychometrically sound evaluation methods and outcome instruments, particularly those that can follow pediatric patients through childhood and adolescence; and inadequate funding. ${ }^{11-13}$

A CDE structure that is tailored to pediatric SCI could provide a unified approach for coding procedures used for longitudinal monitoring, standardization for collection of data and enable the comparison of results between and across studies. Much like the work that developed CDEs for pediatric traumatic brain injury (TBI) ${ }^{14}$ the purpose of this project was to review the NINDS adult SCI CDE for relevance to children and youth, and to make recommendations for a CDE structure for pediatric SCI while maintaining the integrity of the adult SCI CDE structure to the greatest extent possible when appropriate (Materials and methods section).

\section{APPROACH TO PEDIATRIC SCI CDE DEVELOPMENT}

The approach to the pediatric SCI CDE development was built upon the adult SCI CDE structure ${ }^{1}$ and other pediatric CDE structures. ${ }^{2}$ Specifically, a working group (WG) of professionals representing a broad expertise in research and care of youth with SCI reviewed every adult SCI CDE for relevance to pediatric SCI. The CDEs from the pediatric TBI, Duchenne and Becker muscular dystrophy, congenital muscular dystrophy, spinal muscular atrophy and myotonic dystrophy structures were also reviewed, and the relevant ones were incorporated into the pediatric SCI CDE structure. All relevant pediatric CDEs were intentionally included to support future comparative studies and pooling of the data across diagnostic categories. In addition, new CDEs specific to pediatric SCI were created, if needed.

Four guiding principles were used for selection and creation of the pediatric data elements. The first principle was to align the approach, methods, framework and terminology with the overall NINDS CDE project $^{1}$ and the CDE structure for adult SCI. ${ }^{3}$ Second, pediatric-onset SCI was viewed as a heterogeneous population, primarily because of the inherent growth and development of children. For this reason, aside from three exceptions (Table 1), all of the elements were reviewed, evaluated and modified/created for four age groups: $0-5$ years, 6-12 years, $13-15$ years and $16-18$ years. These age groups were selected based on the recommendations set forth by DeVivo et al. ${ }^{15}$ The third guiding principle was that whenever possible results of published research studies were used to guide recommendations. In the absence of empirical support, clinical practice guidelines and protocols were reviewed, and international content expert consensus was garnered through an iterative process of review, discussion and revision. In addition to producing evidence-based recommendations for pediatric SCI CDEs, this process also enabled us to identify gaps in knowledge and priorities for pediatric SCI research. Last, new CDEs were developed, only under two conditions. For adult CDEs, which were not appropriate for pediatrics, an attempt was made to identify pediatric CDEs that were equivalent; this was most frequently needed in 'Functional Outcome Measures', 'Quality of Life and Participation', 'Alcohol and Tobacco' and 'Sleep'. In addition, new CDEs were developed for areas that were viewed as being highly relevant for pediatric SCI but not relevant for adult-onset SCI, such as birth injuries, school and educational aspects.

To remain consistent with the structure of the NINDS CDE project, the pediatric recommendations were classified as 'Core,' 'Supplemental-Highly Recommended,' 'Supplemental' or 'Exploratory', as defined below:

\section{Core}

This is a data element for recording essential information applicable to any SCI study including all therapeutic areas and study designs. Consistent with all NINDS disease-specific CDE sets, the core pediatric SCI CDEs are a small subset of the available CDEs that are required for all compliant pediatric studies.

\section{Supplemental: highly recommended}

This is a data element that is recommended for use whenever applicable, based on certain conditions or clinical study designs. In most cases, these have been used and validated with strong psychometrics for use in SCI, and are considered essential for clinical research studies by experts in the field.

\section{Supplemental}

This is a data element that has some evidence of validity and is commonly collected in clinical studies in SCI. Its use depends upon the study design, protocol or type of research involved. These are recommended, but not required, for studies, as appropriate. 


\section{Exploratory}

This is a data element that could be emerging or that requires further validation in target populations, but may fill current gaps in the CDEs and/or substitute for an existing CDE with additional evidence.

\section{WORKGROUP CONSISTENCY AND REVIEW PROCESS}

NINDS invited the two primary authors (MJM and LCV) to co-chair the workgroup. Pediatric SCI expert clinicians and researchers were identified through the pediatric committee of the American Spinal Injury Association, membership of the International Spinal Cord Society and Pan European Pediatric SCI Collaboration and attendees of past Howard H. Steel Conferences on Pediatric Spinal Cord Injury and Dysfunction, and were invited to participate in the Pediatric SCI WG. WG members also identified additional experts who were subsequently asked to participate. The WG consisted of 13 pediatric experts from six countries - the United States, Australia, Denmark, Switzerland, Spain and Sweden-and three representatives from the NINDS CDE project.

The WG was divided into 15 smaller subgroups, and each was assigned domains from the adult SCI CDE. A leader was identified for each subgroup and was responsible for working with the subgroup members to review the assigned NINDS SCI adult CDEs for relevance to each of the four pediatric age groups, to provide a rationale and support for any changes or additions and to ensure inclusion of disciplines that were not adequately represented in the core workgroup (for example, nursing, nutrition, recreation therapy and radiology).

For each subgroup, a reporting timeline agenda was developed by the WG chairs and used for reporting during weekly or biweekly telephone conferences for seven months (February to September 2015). Workgroup members responded to the review and discussed recommendations. NINDS SCI CDE Team representatives provided background and rationale for the adult SCI CDE, as needed, and directed attention to existing CDEs for other pediatric conditions, as appropriate. If any $\mathrm{CDE}$ recommendation did not reach consensus by the WG, subgroup leaders were asked to further evaluate the recommendation, identify additional supporting evidence (peer review

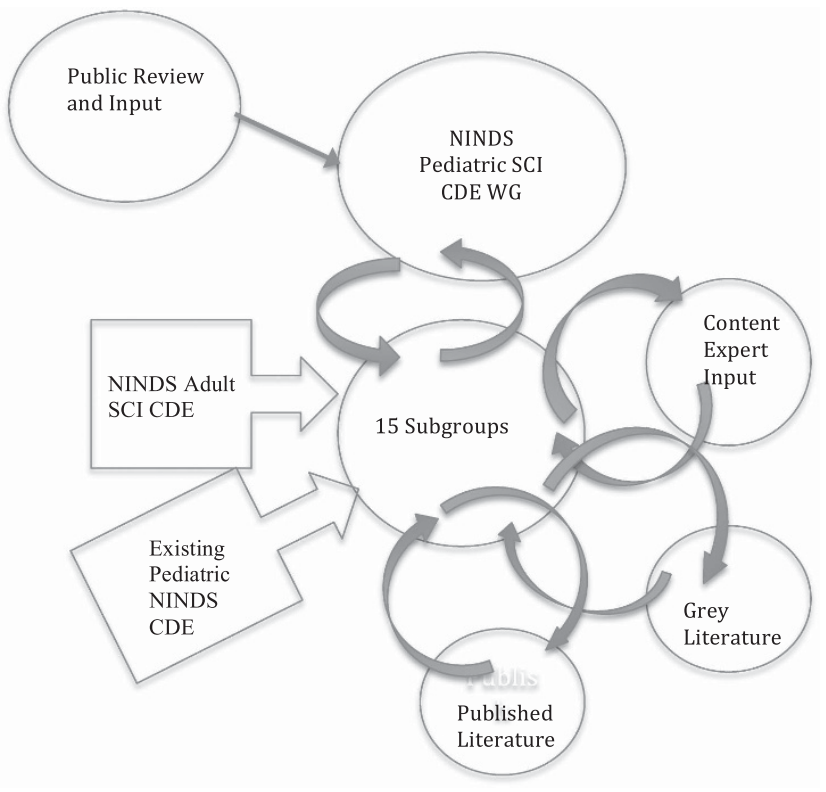

Figure 1 Iterative process for reviewing and modifying the NINDS adult SCI CDEs for relevance to children and youth with $\mathrm{SCl}$. A full color version of this figure is available at the Spinal Cord journal online. publications and grey literature) and/or seek additional external content experts' review while the $\mathrm{CDE}$ was placed back on the reporting timeline agenda for the next conference call. As illustrated in Figure 1, this iterative process was followed for every CDE until all WG members agreed on each $\mathrm{CDE}$ recommendation.

\section{INTERNAL AND PUBLIC REVIEW PROCESSES}

Once the WG completed the recommendations, the NINDS SCI CDE Team developed and/or modified where necessary all pertinent draft documents. These documents included revised or new CDEs, CRFs and instrument recommendations. The draft of the end product that includes the data dictionary of elements, template CRFs, recommendations for use of standardized instruments/measure, guidelines and summaries for each domain was first reviewed internally by the WG.

After 1 month of review by the WG, the CDEs were released for public review by being posted on the NINDS CDE website for 5 weeks and advertised through e-mail lists and national presentations. The feedback received from public review was incorporated into the final product for version 1.0 of the pediatric CDEs.

Dr Lyn Jakeman and Ms. Odenkirchen receive employment compensation from NIH/NINDS. Ms Sheikh receives employment compensation from Emmes Corporation. None of the other authors have anything to disclose.

\section{RESULTS}

\section{Patient characteristics}

For both the SCI Core and SCI demographic CRFs, explicit instructions were given to advise end-users that the data elements on these CRFs reflect child characteristics, not caregiver characteristics. Additional pediatric variables were added to the SCI demographic CRF to capture educational level and other relevant variables (Table 2). To remain consistent with other pediatric CDEs and to facilitate comparison of study results across diagnostic samples, additional relevant pediatric elements were obtained from the NINDS pediatric TBI and muscular dystrophy CDE sets.

\section{Participant and family history}

As summarized in Table 2, the adult SCI CRF and questionnaires for epidemiology and environmental history were considered 'not appropriate' for children younger than 12 years. In addition, the Alcohol, Tobacco Use and Substance Use CRFs were recommended as Supplemental only for youth 12 years of age and older. There was consensus that the questions on these CRFs should be asked to the child, without the presence of the parent; this consensus is supported by studies showing that children will respond with more honesty when asked questions about alcohol and substance use in the absence of the parent or caregiver. ${ }^{16}$ Two instruments from the adult CDE were classified for subsets of youth. The Alcohol Use Disorders Identification Test Self-Report Version (AUDIT) was recommended as Supplemental for children 14 years and older and the Food Frequency Questionnaire was recommended as Exploratory for youth aged 18-21 years. Three pediatric dietary instruments were recommended as either Exploratory or Supplemental. ${ }^{17-19}$ Given the importance of healthy lifestyle choices on mitigating complications after SCI, the void in research literature and lack of psychometrically sound instruments for evaluation of substance use and dietary habits in children and youth with SCI, further research in this area is recommended.

\section{Disease and injury related events}

Additional Supplemental pediatric-specific elements were added to the SCI Pre-Hospital assessment CRF. Specifically, questions were added 
Table 2 Pediatric recommendations for participant characteristics and participant and family history

\begin{tabular}{|c|c|c|c|}
\hline \multicolumn{3}{|c|}{ Adult $S C I C D E$} & \multirow[t]{2}{*}{ Pediatric recommendations } \\
\hline CDE domain & SCl subdomain & CRF/NOC & \\
\hline \multirow[t]{2}{*}{$\begin{array}{l}\text { Participant } \\
\text { characteristics }\end{array}$} & Demographics & Adult SCl core CRF & $\begin{array}{l}\text { Added instructions: For pediatric studies, the data elements on this CRF reflect child characteristics, } \\
\text { not caregiver characteristics }\end{array}$ \\
\hline & & $\begin{array}{l}\text { Adult SCl demographics } \\
\text { CFR }\end{array}$ & $\begin{array}{l}\text { Pediatric-specific variables added: Genotypic sex; age; level of education; primary caregiver level of } \\
\text { education; participant/subject school status; caregiver marital/partner status; preinjury hand } \\
\text { preference; current hand preference; birthplace; and primary language } \\
\text { Changed recording of age for children }<3 \text { years: if }<3 \text { years of age, define age in months } \\
\text { Added instructions: For pediatric studies, the data elements on this CRF reflect child characteristics, } \\
\text { not caregiver characteristics (unless otherwise specified) }\end{array}$ \\
\hline \multirow[t]{7}{*}{$\begin{array}{l}\text { Participant history } \\
\text { and family history }\end{array}$} & $\begin{array}{l}\text { Epidemiology/ } \\
\text { environmental } \\
\text { history }\end{array}$ & $\begin{array}{l}\text { Alcohol and tobacco use } \\
\text { CRF }\end{array}$ & $\begin{array}{l}\text { Data elements on this CRF are classified as Supplemental for ages } 12 \text { and older. Not appropriate for } \\
\text { younger than } 12 \text { years }\end{array}$ \\
\hline & & AUDIT self-report version & Define as Supplemental for ages 14 and older. Not appropriate for younger than 14 years \\
\hline & & Substance use CRF & $\begin{array}{l}\text { Data elements on this CRF are classified as Supplemental for ages } 12 \text { and older. Not appropriate for } \\
\text { younger than } 12 \text { years }\end{array}$ \\
\hline & & FFQ & Defined as Exploratory for ages 18 and older. Not appropriate for younger than 18 \\
\hline & & YAQ & Added and recommended as: Supplemental for youth, ages 9-21 \\
\hline & & CADET & Added and recommended as: Exploratory for children, ages 3-7 \\
\hline & & $\begin{array}{l}\text { Calcium Counts Food } \\
\text { Frequency Questionnaire }\end{array}$ & Added and recommended as: Exploratory for ages 7-10 \\
\hline
\end{tabular}

Abbreviations: AUDIT, alcohol use disorders identification Test; CADET, child and diet evaluation tool; CDE, common data element; COPM, Canadian occupational performance measure; CRF, case report form; FFQ, Food Frequency Questionnaire; GRT, Grasp and Release Test; NOC, notice of copyright; QIF, Quadraplegia Index of Function; SCI, spinal cord injury;

SCIM, Spinal Cord Independence Measures; YAQ, Youth/Adolescent Questionnaire.

Table 3 Modifications to the assessments and examination subdomains: hospital care/management, physical examination, vital signs and laboratory tests, and imaging

\begin{tabular}{|c|c|c|c|}
\hline \multirow[t]{7}{*}{$\begin{array}{l}\text { Assessments and } \\
\text { examinations }\end{array}$} & Hospital care/management & $\begin{array}{l}\text { Acute admission discharge } \\
\text { CRF } \\
\text { Rehabilitation admission } \\
\text { discharge CRF }\end{array}$ & $\begin{array}{l}\text { Pediatric-specific variables added: Specification of type of ICU (adult ICU, } \\
\text { pediatric ICU, ICU and unspecified) } \\
\text { Pediatric-specific variables added: Rehabilitation facility type (general adult, } \\
\text { general pediatric, SCl adult, SCI pediatric, other and unknown) }\end{array}$ \\
\hline & Physical examinations & Clinical assessment CRF & $\begin{array}{l}\text { Added response option 'Not Applicable/Too Young to Determine' for the } \\
\text { following elements: awareness of the need to empty the bladder; awareness of } \\
\text { the need to defecate; self-reported impact on quality of life due to bowel } \\
\text { dysfunction } \\
\text { Added element: Surgical procedures on the urinary tract } \\
\text { Added element: Problems with swallowing }\end{array}$ \\
\hline & & SCIPUS & Not recommended for children younger than 18 years \\
\hline & & The Braden Q Scale & Added and recommended as supplemental for children $\leqslant 8$ years \\
\hline & & $\begin{array}{l}\text { Swallowing Disturbance } \\
\text { Questionnaire }\end{array}$ & Not recommended for children younger than 18 years \\
\hline & Vital signs and laboratory tests & Laboratory test CRF & Added variable: 25 -hydroxy Vitamin D \\
\hline & $\begin{array}{l}\text { Spinal imaging/spinal cord } \\
\text { imaging guidelines }\end{array}$ & SCI Imaging Guidelines & $\begin{array}{l}\text { Added consideration in instructions: Children under } 7 \text { require sedation, risks } \\
\text { and benefits of research studies need to be considered }\end{array}$ \\
\hline
\end{tabular}

Abbreviations: CRF, case report form; ICU, intensive care unit; SCI, spinal cord injury; SCIPUS, Spinal Cord Injury Pressure Ulcer Scale.

to document the presence of a hypotensive episode; cardiac arrest; Pediatric Glasgow Coma Scale; type of transport to the hospital; and use of a modified backboard for transport (for children 0-5 years). To collect information on injuries sustained in motor vehicle crashes, questions on the use of protective devices (helmet, child safety restraint, set belt, airbag and other); type of restraint used (rear-facing car seat, forward facing car seat and booster seat); type of seat belt (lap, three-point, five-point and unknown); and position in vehicle (front seat, back seat and other) were added. The Pediatric Glasgow Scale was recommended for children younger than 18 years of age. ${ }^{20}$ Finally, a CRF specific to birth-related SCI was developed, and classified as being
'Supplemental-Highly Recommended' for studies involving birth injuries.

\section{Assessments and examinations}

Table 3 summarizes the recommendations for pediatric elements for the assessments and examinations domain. For the acute and rehabilitation admission/discharge CRFs, specification on type of intensive care unit and rehabilitation facility were added. Three modifications were made to the clinical assessment CRF; a response option of 'not applicable' was added for several elements, and two additional elements were added (surgery on urinary tract and 
Table 4 Pediatric recommendations for the outcome and end points: neurological outcomes and electrodiagnostics subdomains

\begin{tabular}{|c|c|c|c|c|}
\hline \multirow[t]{2}{*}{ Subdomain } & \multirow[t]{2}{*}{ Tests and measures } & \multicolumn{2}{|c|}{$\begin{array}{l}\text { NINDS workgroup } \\
\text { recommendations }\end{array}$} & \multirow[t]{2}{*}{ Comment } \\
\hline & & Adult & Pediatric & \\
\hline \multirow[t]{7}{*}{ Neurological outcomes } & ISNCSCI & $\mathrm{C}$ & C & For $\geqslant 6$ years \\
\hline & Modified Ashworth Scale & S & $E$ & Reliability in pediatrics adequate \\
\hline & Tardieu Scale & $E$ & $E$ & No studies in children \\
\hline & SCATS & $E$ & $E$ & No studies in children \\
\hline & $\begin{array}{l}\text { NINDS Myotatic Reflex } \\
\text { Scale }\end{array}$ & $\mathrm{E}$ & $\mathrm{E}$ & No studies in children \\
\hline & $\begin{array}{l}\text { Penn Spams Frequency } \\
\text { Scale }\end{array}$ & $\mathrm{E}$ & $\mathrm{E}$ & No studies in children, requires research as a parent-PRO \\
\hline & Pendulum test & $\mathrm{E}$ & $\mathrm{E}$ & No modification needed in children age 30 months and older \\
\hline \multirow[t]{7}{*}{ Electrodiagnostics } & Peripheral nerve studies & S-HR, S & S-HR, S & $\mathrm{S}-\mathrm{HR}=$ nerve conduction studies; $\mathrm{S}=\mathrm{H}$-reflex, $\mathrm{EMG}$ children may require shorten exam \\
\hline & $\begin{array}{l}\text { Quantitative sensory } \\
\text { testing }\end{array}$ & $\mathrm{S}$, & $\mathrm{S}$ & 8 years and older \\
\hline & $\begin{array}{l}\text { Electrical perceptual } \\
\text { threshold }\end{array}$ & $\mathrm{S}$ & $\mathrm{E}$ & $\begin{array}{l}\text { Younger children will not be able to participate in response; lower age limit may be between } \\
8 \text { and } 10 \text { years. These elements are not used widely in children }\end{array}$ \\
\hline & Sensory evoked potentials & $\mathrm{S}$ & $\mathrm{S}$ & \\
\hline & Motor evoked potentials & S & $\mathrm{S}$ & \\
\hline & $\begin{array}{l}\text { Brain motor control } \\
\text { assessment }\end{array}$ & S & $\mathrm{E}$ & $\begin{array}{l}\text { Younger children will not be able to participate in response; lower age limit may be between } \\
8 \text { and } 10 \text { years }\end{array}$ \\
\hline & $\begin{array}{l}\text { Sympathetic skin } \\
\text { responses }\end{array}$ & S & $\mathrm{S}$ & \\
\hline
\end{tabular}

Abbreviations: EMG, electromyography; ISNCSCI, International Standards for Neurological Classification of Spinal Cord Injury; NINDS, National Institute of Neurologic Disorders and Stroke; PRO, patient reported outcome.

The majority of test and measures for neurologic and electrodiagnostic outcomes for adult and pediatric studies had similar classification. Classification codes: Core (C), Supplemental (S),

Supplemental-Highly Recommended (S-HR) and Exploratory (E).

problems with swallowing; Table 3). Because there are no psychometric studies in children and they are not used in pediatric SCI practices, the Spinal Cord Injury Pressure Ulcer Scale and Swallowing Disturbance Questionnaire were not recommended for youth younger than 18 years of age. The Braden Q Scale was recommended as Supplemental for children younger than 8 years of age. ${ }^{21}$ The availability of psychometrically sound assessments for pressure ulcer prediction and risk was identified as a gap ${ }^{22}$ and research in this area was recommended, with high priority.

The existing adult SCI Laboratory Tests CRF was considered relevant and appropriate for use with children. One element was added (25-hydroxy vitamin D) and classified as 'Supplemental-Highly Recommended' for both children and adults. ${ }^{23,24}$ No changes were made to the Spinal Imaging CRF or guidelines. Consideration about the benefits and risks of imaging studies in very young children who require sedation for optimal imaging was added to the guidelines.

\section{Treatment/intervention data domain: therapies domain}

Research in the rehabilitation domain is wide-ranging, and it represents a rapidly changing area with particular complexity of data collection depending on the environment and the goals of individual studies. As a starting point for recommendations in studies for youth with SCI, the Rehabilitation Therapies CRF developed for adult SCI was reviewed and revised with both pediatric modifications and overall changes for ease of use. Options for both adult and pediatric participants were added for the rehabilitation services received and follow-up care data elements. The existing chart for collection of data on rehabilitation services received was modified for clarity. The table for types of therapy was revised with the additions of child-life therapy, art, music or play therapy, exercise physiology/kinesiology and personal trainer. Similarly, the pediatric WG added school/ educational reintegration outing, play education, caregiver/attendant education, serial casting and hippotherapy to the activities table. The data element for mobility devices was removed and expanded as the new assistive/mobility devices and orthoses CRF, with additional permissible values for wheelchairs, walkers and different types of orthoses and standers. A separate question to collect data on upperextremity orthoses was added to this form, as well as a question on body jacket/back brace/TLSO, with specific relevance to neuromuscular scoliosis.

The pediatric WG also created a falls diary CRF for SCI, which includes a table where falls, as well as questions pertaining to any details on specific falls, can be recorded. The questions capture information including type of footwear worn, circumstances when the fall happened, assistive devices being used and any injuries sustained.

Outcome and end points: neurological and electrodiagnostics Table 4 summarizes the recommendations for the neurological and electrodiagnostic CDEs. Owing to the void in psychometric research in children with SCI, none of the adult measures for neurological outcomes were considered developed for children and were therefore classified as Exploratory, with the exception of the International Standards for Neurological Classification of Spinal Cord Injury (ISNCSCI). Similarly, there were no pediatric-specific instruments for neurological outcomes or spasticity with psychometric support that could be recommended as alternatives to the adult instruments. Thus, research on validating existing or developing new instruments that 
evaluate neurologic end points was highly recommended. The ISNCSCI was recommended as Core for children aged 6 years and older based on several large-scale pediatric psychometric studies. ${ }^{25-27}$ An alternative to the ISNCSCI for children younger than 6 years old is a high priority for research.

The pediatric recommendations for electrodiagnostic outcomes were based primarily on clinical experience and protocols. As shown in Table 4, pediatric recommendations for electrodiagnostic outcomes closely resemble the ones made for adult studies, but because reliability of values in children is not established, their use as primary end points in pediatric clinical trials is not recommended.

Table 5 Pediatric recommendations for functional outcomes test and measures identified in adult $\mathrm{SCl}$

\begin{tabular}{|c|c|c|c|}
\hline \multirow[t]{2}{*}{ Test and measures } & \multicolumn{2}{|c|}{$\begin{array}{l}\text { NINDS workgroup } \\
\text { recommendations }\end{array}$} & \multirow[t]{2}{*}{ Comments } \\
\hline & Adult & Pediatric & \\
\hline 10- min timed walk & S-HR & S & For age $\geqslant 2$ years \\
\hline 2 -min walk test & $\mathrm{S}$ & $E$ & For age $4-18$ years \\
\hline 6 -min walk test & S-HR & S & For age $\geqslant 4$ \\
\hline Berg Balance Scale & S-HR & NR & For $<18$ years \\
\hline Borg RPE Scale & $\mathrm{E}$ & $E$ & For age $\geqslant 10$ years \\
\hline COPM & $\mathrm{S}$ & S & $\begin{array}{l}\text { For } \geqslant 2 \text { years as parent report and for } \\
\geqslant 6 \text { years for child report }\end{array}$ \\
\hline CUE-Q & S & $S, E$ & $S$ for $\geqslant 16$ years, $E$ for $6-15$ \\
\hline CUE-T & $E$ & $E$ & For age $\geqslant 16$ \\
\hline $\begin{array}{l}\text { Five-time sit to stand } \\
\text { test }\end{array}$ & $\mathrm{S}$ & S & For age $\geqslant 4$ years \\
\hline GRASSP & $\mathrm{E}$ & $E$ & For $\geqslant 5$ years \\
\hline GRT & $E$ & $E$ & For $\geqslant 7$ years \\
\hline $\begin{array}{l}\text { Jebsen taylor hand } \\
\text { function Test }\end{array}$ & $\mathrm{S}$ & S & For $+>6$ years \\
\hline $\begin{array}{l}\text { Neuromuscular } \\
\text { Recovery Scale }\end{array}$ & $\mathrm{E}$ & $E$ & For $\geqslant 12$ years \\
\hline Nine hole peg test & $E$ & $E$ & For $\geqslant 4$ years \\
\hline QIF & $E$ & NR & For $<18$ years \\
\hline $\begin{array}{l}\text { Sollerman test of hand } \\
\text { function }\end{array}$ & $\mathrm{E}$ & NR & For $<18$ years \\
\hline SCIM-III & S-HR & $\mathrm{E}, \mathrm{S}$ & $E$ for up to 8 years, $S$ for $\geqslant 8$ years \\
\hline SCIM-III-SR & S-HR & NR & $\begin{array}{l}\text { SCIM-III SR required extensive } \\
\text { modification for child self-report }\end{array}$ \\
\hline SCI-FAI & $\mathrm{S}$ & $E$ & For $2-17$ years \\
\hline $\mathrm{SCl}-\mathrm{FI}$ & $E$ & NR & For $<18$ years \\
\hline Stair climb & $\mathrm{S}$ & S & For age $\geqslant 4$ years \\
\hline $\begin{array}{l}\text { Stride analysis and gait } \\
\text { variability }\end{array}$ & $\mathrm{S}$ & S & $\begin{array}{l}\text { For age }+>3 \text {; intra-subject analysis is } \\
\text { recommended because of lack of } \\
\text { pediatric norms }\end{array}$ \\
\hline THAQ & $\mathrm{E}$ & $E$ & For $+>12$ years \\
\hline The ABLE Scale & $E$ & NR & For $<18$ years \\
\hline TUG & $\mathrm{S}$ & $\mathrm{S}$ & For $\geqslant 3$ years \\
\hline $\begin{array}{l}\text { Walking ilndex for } \\
\text { SCI-II }\end{array}$ & $\mathrm{S}$ & S & For $+>3$ years \\
\hline Wheelchair circuit & $\mathrm{E}$ & $E$ & For $\geqslant 5$ years \\
\hline Wheelchair skills test & $\mathrm{S}$ & $S, E$ & $S$ for $\geqslant 6$ years, $E$ for $<6$ years \\
\hline
\end{tabular}

Abbreviations: ABLE Scale, activity-based balance level evaluation scale; Borg RPE Scale, Borg rating of perceived exertion scale; COPM, Canadian occupational performance measure E, exploratory; NINDS, National Institute of Neurologic Disorders and Stroke; NR, not recommended; RPE, rating of perceived exertion;

S, supplemental; S-HR, supplemental-highly recommended; $\mathrm{SCl}$, spinal cord injury; THAQ, Tetraplegia Hand Activity Questionnaire; TUG, times up and go.

\section{Outcome and end points: functional outcomes}

Of the 28 functional outcomes instruments included in the adult SCI CDE set, five were identified as 'not appropriate' for youth younger than 18 years of age (Table 5). Ten instruments were classified as 'Supplemental' and the remaining 13 instruments were classified as 'Exploratory' (Table 5). With the exception of the WISCI-II, which has been field-tested in children with $\mathrm{SCI},{ }^{28}$ instruments that focused on walking and upright mobility were classified based on the age when walking is typically achieved ( 18 months -3 years), the availability of normative values and psychometric studies with children with cerebral palsy and other physical conditions. Similarly, there were few upper-extremity instruments that had pediatric psychometric support. The Jebsen Taylor Test of Hand Function was classified as 'Supplemental' for children 6 years of age and older, based on normative values and studies with several clinical samples, but not SCI. Five other upper-extremity (UE) instruments were classified as 'Exploratory' (Table 5).

The majority of pediatric functional outcome instruments from other NINDS pediatric CDE structures (Table 6) were classified as 'Exploratory', as they lacked psychometric studies with children with SCI; the exceptions were the Pediatric Evaluation of Disability Index $(\mathrm{PEDI})^{29}$ and the Functional Independence Measure for Children (WeeFIM). ${ }^{30}$ Three pediatric SCI-specific instruments ${ }^{31-35}$ that have recently been developed and field-tested were also recommended as 'Exploratory'. Developing new and/or validating existing relevant functional outcome measures for pediatric SCI was identified as a high priority for research.

\section{Outcome and end points: pain}

None of the pain instruments recommended for adult SCI were considered appropriate for children and youth. Four pediatric-specific instruments were identified for the assessment of pain and were classified as 'Supplemental'. The Functional Disability Inventory-Child and Adolescent Form ${ }^{36}$ is the most common functional instrument for pediatric pain populations. The Adolescent Pediatric Pain Tool ${ }^{37}$ and Children's Activity Limitation Interview ${ }^{38}$ were recommended for children aged 8-17 and 8-18 years, respectively. The Revised Faces Pain Scale ${ }^{39}$ was recommended for children between 4 and 8 years of age.

\section{Outcome and end points: participation and quality of life}

None of the adult participation or quality-of-life (QOL) measures were considered appropriate for children. Five pediatric-specific instruments were identified for the assessment of participation and QOL. The Child Health Questionnaire ${ }^{40}$ was classified as 'Supplemental' because of the availability of norm references, standardization of testing, scoring and interpretation, and psychometric testing in pediatric clinical samples. It is a measure of quality of life, and is available as a parent report (aged 5-10 years) and child report (older than 10 years). The pediatric versions of the EuroQOL-5 Dimension Questionnaire (EQ-5DY) ${ }^{41}$ and the Satisfaction with Life Scale for Children (SWLS-C) ${ }^{42}$ were classified as 'Supplemental' for children aged between 8 and 16 years and older than 10 years, respectively. The Child and Adolescent Scale of Participation $(\mathrm{CASP})^{43}$ was developed for children with TBI and classified as 'Supplemental' in the NINDS pediatric TBI and mitochondrial disease CDE sets; given the lack of psychometric support in youth with SCI, it was classified as 'Exploratory' for pediatric SCI. The Pediatric Measure of Participation (PMoP) is a relatively new measure, ${ }^{44,45}$ designed specifically for pediatric SCI, and it was classified as Exploratory. 
Table 6 New pediatric-specific tests and measures of functional outcome

\begin{tabular}{|c|c|c|c|}
\hline Test and measures & Classification pediatric $\mathrm{SCl}$ & Classification other pediatric CDE structures & Comment \\
\hline Peabody Developmental Motor Scale & $\mathrm{E}$ (age $0-5$ years) & $\begin{array}{l}\text { S- HR Mito } \\
\text { S- TBI }\end{array}$ & No studies in pediatric $\mathrm{SCl}$ \\
\hline PEDI; PEDI CAT & S-HR (age 6 months-7 years) & $\begin{array}{l}\text { B- TBI acute } \\
\text { S- TBI chronic } \\
\text { S- CMD } \\
\text { E- DMD }\end{array}$ & \\
\hline $\begin{array}{l}\text { Pediatric Neuromuscular } \\
\text { Recovery Scale }\end{array}$ & $\mathrm{E}$, (age $1-12$ years) & NA & $\begin{array}{l}\text { Based on modifications of the adult } \\
\text { Neuromuscular Recovery Scale }\end{array}$ \\
\hline PEDI-SCI AM CAT and SF & $\begin{array}{l}\text { E, (age } 4-21 \text { years for parent report; } \\
8-21 \text { years for child report) }\end{array}$ & NA & \\
\hline SATCO & $\mathrm{E}$, (age $0-18$ years) & NA & \\
\hline SCIM-III SR-Y & $\mathrm{E},(+>8$ years $)$ & NA & Based on modifications of the SCIM-III SR \\
\hline Bayley Scale & E, (age 1-42 months) & $\begin{array}{l}\text { S-HR- CMD } \\
\text { S- Epilepsy, Mito, NMD, SMA, Stroke and } \\
\text { TBI }\end{array}$ & No studies in pediatric $\mathrm{SCl}$ \\
\hline BOT-2 & $E$, (age $4-12$ years) & S- TBI & No studies in pediatric $\mathrm{SCl}$ \\
\hline PROMIS- Peds & $E$ & NA & No studies in pediatric $\mathrm{SCl}$ \\
\hline GMFM-88; GMFM-66 & $\mathrm{E}$, (age 5 months -16 years) & $\begin{array}{l}\text { S- TBI, SMA, DM, Mito } \\
\text { E- CMD, DMD }\end{array}$ & No studies in pediatric $\mathrm{SCl}$ \\
\hline WeeFIM & S, (age $0-7$ years) & S- TBI & \\
\hline
\end{tabular}

Abbreviations: BOT-2, Bruininks-Oseretsky test of motor proficiency-2; CAT, computerized adaptive test; CMD, congenital muscular dystrophy; DMD, duchene muscular dystrophy; E, exploratory; GMFM, gross motor functional measure; Mito, Mitochondria disease; NA, not applicable; NMD, neuromuscular disease; NR, not recommended; Peds, pediatric; PEDI, pediatric evaluation of disability inventory; PROMIS, patient reported outcome measurement information system; S, supplemental; S-HR, supplemental-highly recommended; SATCo, segmental assessment of trunk control; SCIM-III SR-Y, spinal cord independence measure-III self report for youth; SMA, spinal muscle atrophy; SF, short form; TBI, traumatic brain injury; WeeFIM, functional independence control; SCIM-III SR-Y, spinal cord
measure for children; yrs, years.

Table 7 Pediatric recommendations for tests and measures for psychological status and sleep subdomains

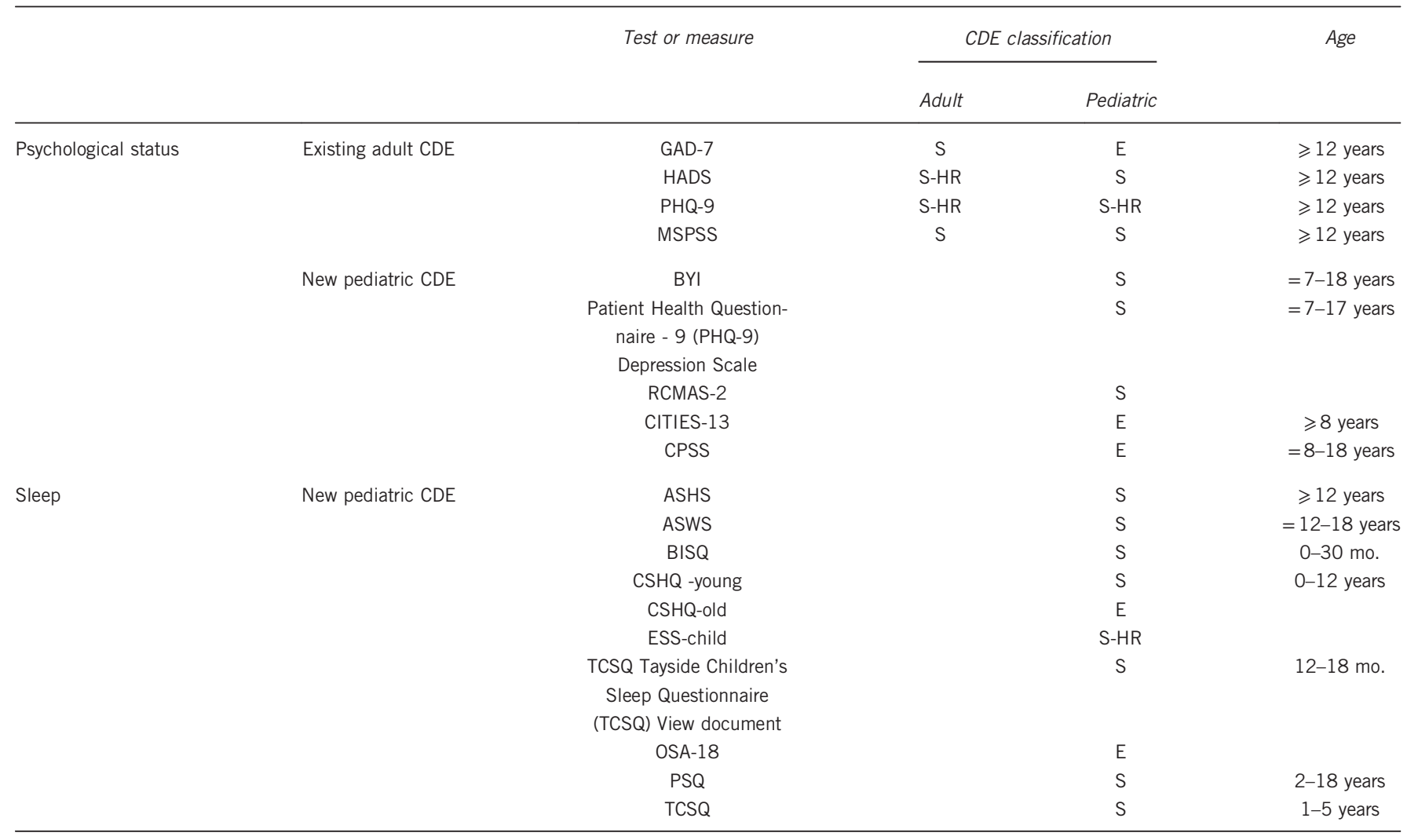




\section{Outcome and end points: psychological status and sleep}

Of the eight adult SCI psychological status instruments, four were recommended for children aged 12 years and older (Table 7). Five pediatric-specific instruments were identified for the assessment of psychological status (Table 7) and classified as 'Exploratory ${ }^{46,47}$ and 'Supplemental. ${ }^{48-50}$ The International Classification of Sleep Disorders (ICSD) Criteria, which is classified as 'Supplemental' for adult SCI, was also recommended as 'Supplemental' for pediatric SCI, and the Pittsburgh Sleep Quality Index (PSQI), which is classified as 'Exploratory' for adult SCI, was also recommended as 'Exploratory' for pediatric SCI. Nine additional instruments that evaluate sleep in pediatrics were recommended and, with the exception of one, ${ }^{51}$ classified as 'Supplemental' ${ }^{52-59}$ (Table 7).

\section{Integration of comments from public review}

Public feedback and all comments were compiled by the NINDS CDE team and shared with the pediatric WG. The group addressed each concern or suggested edits as appropriate. Once a consensus was achieved, the recommended changes were made and confirmed with the group before finalizing documents for posting on the NINDS CDE website.

\section{The international data sets}

Although selected sets and individual CDEs from the International SCI data sets are integrated throughout the NINDS SCI CDEs, the full list of the International SCI Data Sets ${ }^{8}$ was not comprehensively reviewed by the NINDS pediatric SCI WG. As the steering committee of the International SCI Data Sets conducts their ongoing formal review of the International SCI Data Sets, the pediatric CDE recommendations will be integrated into the modifications and posted for public review before being finalized.

\section{DISCUSSION}

Iterative review of the adult SCI NINDS CDEs by content experts and use of empirical studies, clinical practice guidelines, practice protocols and existing CDEs for pediatric conditions other than SCI formed the basis for recommendations for CDEs for clinical studies of pediatric SCI. These recommendations were provided for public review before being endorsed and made available by NINDS and are meant to facilitate pediatric SCI research and data sharing, through the use of CRFs and common tests, instruments and outcome measures. Furthermore, the international composition of the pediatric WG helped to ensure global applicability of the CDEs.

As a publically available resource, the widespread use of the pediatric SCI CDEs will help facilitate the sharing of pediatric SCI clinical research data across a broad range of studies worldwide. All recommended CRFs and instruments are listed on the NINDS CDE website and have accompanying guidelines available to select the appropriate CDEs for a particular study. CRFs can be downloaded and used directly from the website; copyrighted instruments have informational documents, which will direct the user on how to obtain the necessary permissions for use. Specific CDEs can also be found using the CDE Search tools on the website. Researchers should be aware that these recommendations do not substitute for consultation by experts in pediatric SCI, and are encouraged to contact the NINDS CDE team with any questions regarding implementation of the CDEs.

This work highlighted the glaring void in well-designed clinical trials and outcome studies in pediatric SCI, and the lack of psychometrically sound instruments for many end points of interest. The pediatric SCI CDEs provide a starting point for researchers with guidelines on the use of CRFs, tests and measures that have been classified as 'Core', 'Supplemental-Highly Recommended' or 'Supplemental', which will reduce research start-up time and costs and provide standardization for data collection protocols. Researchers can also use the classification of instruments to guide priority for psychometric studies; specifically, those instruments classified as 'Exploratory' need further validation studies in children with SCI. Similarly, the WG developed consensus on priorities for research, which may guide study design and funding decisions.

Many of the added CDEs were taken from existing NINDS CDEs for pediatric TBI, duchene muscular dystrophy and mitochondrial disease; yet, there were several unique features of pediatric-onset SCI that required the development of new CDEs. Use of the newly developed CDEs for birth injuries will enable pooling of data on a relatively rare etiology of pediatric SCI. Lap-belt injuries and musculoskeletal complications in children with SCI are highly prevalent, and as a result CDEs for hip instability, neuromuscular scoliosis and lapbelt injuries were developed.

Uptake and implementation of the pediatric SCI CDEs by pediatric researchers should be adopted. Given the small pediatric SCI population, multicenter studies using the CDEs have the potential to achieve a large-enough sample to answer important research questions. Clinical uptake has strong potential to facilitate quality improvement projects, and evidence-based pediatric SCI practice.

The formal review of the NINDS adult SCI CDEs resulted in identification of gaps in knowledge about key areas of pediatric SCI. Priorities for research include development and validation of instruments and outcome measures of functional capacity, upper extremity, pain, sleep, quality of life, participation and neurological outcomes (for children aged 0-5); understanding of needs and priorities of caregivers and siblings; development of evidence-based clinical practice guidelines for electrodiagnostic testing and spinal cord imaging; nontraumatic pediatric SCI; and pediatric validation of the International Data Sets.

One of the greatest research needs relevant to outcome measures is for evaluation and classification of the neurological consequence of SCI in children aged younger than 6 years, as well as development of measures for upper-extremity function, participation and sleep domains. Moreover, with few exceptions, there is a glaring void in outcome instruments that are capable of administration across the broad pediatric-adolescent age span. This void forces the administration of different instruments for different age groups, and is directly responsible for the inability to compare outcomes longitudinally as children age. Addressing this void is a research priority.

Finally, there is a marked void in pediatric SCI standards of practice, suggesting the urgent need for well-designed, multicenter longitudinal studies and clinical trials in this population.

\section{CONCLUSION}

Since 2006, NINDS has led the development of CDEs and guidance documents for use in clinical studies of neurological disorders and diseases. This current effort extends this resource to include recommendations, CDEs, CRFs and data structures for use in studies of SCI in pediatric populations. The pediatric SCI CDEs are incorporated directly into the NINDS SCI CDE sets and can be found at https:// commondataelements.ninds.nih.gov. As with all the SCI CDEs, these will continue to be harmonized with the ISCoS International SCI Data Sets as part of a continual updating and review process. Throughout this process, the pediatric SCI CDE WG identified areas that require further development, including a need for increased research to develop and validate pediatric-relevant outcome measures in a number of domains. Given the very small number of patients in this 
population, the use of CDEs for clinical research is essential for enabling standardized reporting and data sharing across sites and studies to develop and test strategies and interventions for improving recovery and outcomes for children and youth with SCI.

\section{CONFLICT OF INTEREST}

LBJ and JO receive employment compensation from NIH/NINDS. MS receives employment compensation from Emmes Corporation. The remaining authors declare no conflict of interest.

\section{ACKNOWLEDGEMENTS}

We thank Theresa Beck RN; Randal R Betz MD; Loren Davidson MD; Scott Faro MD; Laura Krisa PhD; Feroze Mohamed PhD, Nigel Price MD; Amer Samdani MD; and Colleen Smith.

1 Grinnon ST, Miller K, Marler JR, Lu Y, Stout A, Odenkirchen J et al. National Institute of Neurological Disorders and Stroke Common Data Element Project-approach and methods. Clin Trials (London, England) 2012; 9: 322-329.

2 National Institute of Neurological Disorders and Stroke. NINDS Common Data Elements Database 2006. https://commondataelements.ninds.nih.gov.

3 Biering-Sorensen F, Alai S, Anderson K, Charlifue S, Chen Y, DeVivo M et al. Common data elements for spinal cord injury clinical research: a National Institute for Neurological Disorders and Stroke project. Spinal Cord 2015; 53: 265-277.

4 Biering-Sorensen F, Charlifue S, Devivo MJ, Grinnon ST, Kleitman N, Lu Y et al. Using the spinal cord injury common data elements. Top Spinal Cord Inj Rehabil 2012; 18 23-27.

5 Dvorak MF, Noonan VK, Fallah N, Fisher CG, Rivers CS, Ahn $\mathrm{H}$ et al. Minimizing errors in acute traumatic spinal cord injury trials by acknowledging the heterogeneity of spinal cord anatomy and injury severity: an observational Canadian cohort analysis. J Neurotrauma 2014; 31: 1540-1547.

6 Hawryluk GW, Rowland J, Kwon BK, Fehlings MG. Protection and repair of the injured spinal cord: a review of completed, ongoing, and planned clinical trials for acute spinal cord injury. Neurosurg Focus 2008; 25: E14.

7 Lammertse DP. Clinical trials in spinal cord injury: lessons learned on the path to translation. The 2011 International Spinal Cord Society Sir Ludwig Guttmann Lecture. Spinal Cord 2013; 51: 2-9.

8 Biering-Sorensen F, Charlifue S, Devivo MJ, Grinnon ST, Kleitman N, Lu Y et al. Incorporation of the International Spinal Cord Injury Data Set elements into the National Institute of Neurological Disorders and Stroke Common Data Elements. Spinal Cord. 2011; 49: 60-64.

9 Vogel L, Zebracki K, Betz RR, Mulcahey MJ. (eds). The Child with SCl. McKeith Press London, UK, 2014

10 Zebracki K, Vogel LC. Epidemiology of pediatric onset spinal cord injuries in the United States. In: Rahimi-Movaghar V, Jazayeri SB, Vaccaro AR (eds). Epidemiology of Spinal Cord Injuries. Nova Science Publishers: New York, USA, 2012, pp 19-28.

11 Mulcahey MJ, Sorensen F. Assessment of children with spinal cord injury. In: Vogel LC, Zebracki K, Betz RR, Mulcahey MJ (eds). Spinal Cord Injury in the Child and Young Adult. Wiley: New York, USA, 2015.

12 Parent S, Mac-Thiong JM, Roy-Beaudry M, Sosa JF, Labelle H. Spinal cord injury in the pediatric population: a systematic review of the literature. J Neurotrauma $2011 ; 28$ 1515-1524

13 Vogel LC, Betz RR, Mulcahey MJ. Spinal cord injuries in children and adolescents. Handb Clin Neurol 2012; 109: 131-148.

14 Miller AC, Odenkirchen J, Duhaime AC, Hicks R. Common data elements for research on traumatic brain injury: pediatric considerations. J Neurotrauma 2012; 29 634-638.

15 DeVivo MJ, Biering-Sorensen F, New P, Chen Y. Standardization of data analysis and reporting of results from the International Spinal Cord Injury Core Data Set. Spinal Cord 2011; 49: 596-599.

16 Herrera AV, Benjet C, Mendez E, Casanova L, Medina-Mora ME. How mental health interviews conducted alone, in the presence of an adult, a child or both affects adolescents' reporting of psychological symptoms and risky behaviors. J Youth Adolesc (e-pub ahead of print 20 January 2016; doi:10.1007/s10964-016-0418-1).

17 Christian MS, Evans CE, Nykjaer C, Hancock N, Cade JE. Measuring diet in primary school children aged 8-11 years: validation of the Child and Diet Evaluation Tool (CADET) with an emphasis on fruit and vegetable intake. Eur J Clin Nutr 2015; 69 234-241.

18 Rockett HR, Breitenbach M, Frazier AL, Witschi J, Wolf AM, Field AE et al. Validation of a youth/adolescent food frequency questionnaire. Prev Med 1997; 26 808-816.

19 Zemel BS, Carey LB, Paulhamus DR, Stallings VA, Ittenbach RF. Quantifying calcium intake in school age children: development and validation of the Calcium Counts! food frequency questionnaire. Am J Hum Biol 2010; 22: 180-186.
20 Beers SR, Wisniewski SR, Garcia-Filion P, Tian Y, Hahner T, Berger RP et al. Validity of a pediatric version of the Glasgow Outcome Scale-Extended. J Neurotrauma 2012; 29 . $1126-1139$

21 Curley MA, Razmus IS, Roberts KE, Wypij D. Predicting pressure ulcer risk in pediatric patients: the Braden Q Scale. Nurs Res 2003; 52: 22-33.

22 Kottner J, Hauss A, Schluer AB, Dassen T. Validation and clinical impact of paediatric pressure ulcer risk assessment scales: a systematic review. Int J Nurs Stud 2013; 50: 807-818.

23 Oleson CV, Patel PH, Wuermser LA. Influence of season, ethnicity, and chronicity on vitamin D deficiency in traumatic spinal cord injury. J Spinal Cord Med 2010; 33: 202-213.

24 Nemunaitis GA, Mejia M, Nagy JA, Johnson T, Chae J, Roach MJ. A descriptive study on vitamin $D$ levels in individuals with spinal cord injury in acute inpatient rehabilitation setting. PMR 2010; 2: 202-208.

25 Mulcahey MJ, Gaughan J, Betz RR, Johansen KJ. The International Standards for Neurological Classification of Spinal Cord Injury: reliability of data when applied to children and youths. Spinal Cord 2007; 45: 452-459.

26 Mulcahey MJ, Gaughan JP, Chafetz RS, Vogel LC, Samdani AF, Betz RR. Interrater reliability of the international standards for neurological classification of spinal cord injury in youths with chronic spinal cord injury. Arch Phys Med Rehabil 2011; 92 1264-1269.

27 Samdani A, Chafetz RS, Vogel LC, Betz RR, Gaughan JP, Mulcahey MJ. The International Standards for Neurological Classification of Spinal Cord Injury: relationship between S4-5 dermatome testing and anorectal testing. Spinal Cord 2011; 49: 352-356.

28 Calhoun CL, Mulcahey MJ. Pilot study of reliability and validity of the Walking Index for Spinal Cord Injury II (WISCI-II) in children and adolescents with spinal cord injury. J Pediatr Rehabil Med 2012; 5: 275-279.

29 Choksi A, Townsend EL, Duman HM, Haley SM. Functional recovery in children and adolescents with spinal cord injury. Pediatr Phys Ther 2010; 22: 214-221.

30 Garcia RA, Gaebler-Spira D, Sisung C, Heinemann AW. Functional improvement after pediatric spinal cord injury. Am J Phys Med Rehabil 2002; 81: 458-463.

31 Aldino E, Mulcahey MJ, Trimble S, Argetsinger L, Bienkowski M, Mullen C et al. Development and initial validation of the pediatric neurorecovery scale. Pediatr Phys Ther J 2016; 28: 416-426.

32 Bent L, Mulcahey MJ, Kelly E, Calhoun C, Tian F, Pensheng N et al. Child- and parentreport computer-adaptive tests for assessing daily routines among youth with spinal cord injury. Top Spinal Cord Inj Rehabil 2013; 19: 104-113.

33 Tian F, Pengsheng N, Mulcahey MJ, Hambleton R, Tulsky D, Haley SM et al. Tracking functional status across the spinal cord injury lifespan: linking pediatric and adult patient reported outcome scores. Arch Phys Med Rehabil 2014; 95: 2078-2085.

34 Slavin MD, Mulcahey MJ, Calhoun Thielen C, Ni P, Vogel LC, Haley SM et al. Measuring activity limitation outcomes in youth with spinal cord injury. Spinal Cord 2016; 54: 546-552.

35 Mulcahey MJ, Calhoun C, Vogel L, Kelly E, Sinko. R. The spinal cord independence measure self report for youth. Spinal Cord 2016; 54: 204-212.

36 Claar RL, Walker LS. Functional assessment of pediatric pain patients: psychometric properties of the functional disability inventory. Pain 2006; 121: 77-84.

37 Savedra MC, Tesler MD, Holzemer WL, Wilkie DJ, Ward JA. Pain location: validity and reliability of body outline markings by hospitalized children and adolescents. Res Nurs Health 1989; 12: 307-314.

38 Palermo TM, Witherspoon D, Valenzuela D, Drotar DD. Development and validation of the Child Activity Limitations Interview: a measure of pain-related functional impairment in school-age children and adolescents. Pain 2004; 109: 461-470.

39 Hicks CL, von Baeyer CL, Spafford PA, van Korlaar I, Goodenough B. The Faces Pain Scale-Revised: toward a common metric in pediatric pain measurement. Pain 2001; 93: 173-183.

40 Landgraf JM, Abetz L, Ware jE. CHQ: Child Health Questionnaire. Healthactchq, Inc.: Boston, MA, USA, 2013.

41 Wille N, Badia X, Bonsel G, Burström K, Cavrini G, Devlin N et al. Development of the EQ-5D-Y: a child-friendly version of the EQ-5D. Qual Life Res 2010; 19: 875-886.

42 Gadermann AM, Schonert-ReichI KA, Zumbo BD. Investigating validity evidence of the Satisfaction with Life Scale Adapted for Children. Soc Indic Res 2010; 96: 229-247.

43 Bedell G. Further validation of the Child and Adolescent Scale of Participation (CASP). Dev Neurorehabil 2009; 12: 342-351.

44 Mulcahey MJ, Calhoun CL, Tian F, Ni P, Vogel LC, Haley SM. Evaluation of newly developed item banks for child-reported outcomes of participation following spinal cord injury. Spinal cord 2012; 50: 915-919.

45 Mulcahey MJ, Slavin MD, Ni P, Vogel LC, Theilen CC, Coster WJ et al. The Pediatric Measure of Participation (PMoP) short forms. Spinal Cord 2016; 54: 1183-1187.

46 Foa EB, Johnson KM, Feeny NC, Treadwell KR. The child PTSD Symptom Scale: a preliminary examination of its psychometric properties. J Clin Child Psychol 2001; 30: 376-384.

47 Weiss DS. Impact of Event Scale-revised. In: Wilson JP, Keane TM (eds). Assessing Psychological Trauma and PTSD. 2nd edn Guilford Press: New York, USA, 2004, pp 168-189.

48 Anderson CJ, Kelly EH, Klaas SJ, Russell H, Daharsh E, Vogel LC. Anxiety and depression in children and adolescents with spinal cord injuries. Dev Med Child Neurol 2009; 51: 826-832.

49 Saylor CF, Finch AJ Jr, Spirito A, Bennett B. The children's depression inventory: a systematic evaluation of psychometric properties. J Consult Clin Psychol 1984; 52: 955-967. 
50 Steer RA, Kumar G, Beck AT, Beck JS. Dimensionality of the Beck Youth Inventories with Child Psychiatric Outpatients. J Psychopathol Behav Assess 2005; 27: 123-131.

51 Owens JA, Spirito A, McGuinn M. The Children's Sleep Habits Questionnaire (CSHQ): psychometric properties of a survey instrument for school-aged children. Sleep 2000; 23: 1043-1051.

52 Chervin RD, Hedger K, Dillon JE, Pituch KJ. Pediatric sleep questionnaire (PSQ): validity and reliability of scales for sleep-disordered breathing, snoring, sleepiness, and behavioral problems. Sleep Med 2000; 1: 21-32.

53 Goodlin-Jones BL, Sitnick SL, Tang K, Liu J, Anders TF. The Children's Sleep Habits Questionnaire in toddlers and preschool children. J Dev Behav Pediatr 2008; 29 82-88.

54 McGreavey JA, Donnan PT, Pagliari HC, Sullivan FM. The Tayside children's sleep questionnaire: a simple tool to evaluate sleep problems in young children. Child Care Health Dev 2005; 31: 539-544.
55 Morrell JMB. The infant sleep questionnaire: a new tool to assess infant sleep problems for clinical and research purposes. Child Psychol Psychiatry Rev 1999; 4: 20-26.

56 Sadeh A. A brief screening questionnaire for infant sleep problems: validation and findings for an Internet sample. Pediatrics 2004; 113: e570-e577.

57 Storfer-Isser A, Lebourgeois MK, Harsh J, Tompsett CJ, Redline S. Psychometric properties of the Adolescent Sleep Hygiene Scale. J Sleep Res 2013; 22 707-716.

58 Sufrinko AM, Valrie CR, Lanzo L, Bond KE, Trout KL, Ladd RE et al. Empirical validation of a short version of the Adolescent Sleep-Wake Scale using a sample of ethnically diverse adolescents from an economically disadvantage community. Sleep Med 2015; 16: 1204-1206.

59 Johns MW. A new method for measuring daytime sleepiness: the Epworth sleepiness scale. Sleep 1991; 14: 540-545. 